\title{
Estudio DFT de una sal de imidazolio funcionalizada con quinolina
}

\author{
A DFT study of an imidazolium salt functionalized with quinolone \\ N. Islas-Rodríguez iD a,* O. J. Hernández-Ortiz ${ }^{\text {iDa }}$, R. A. Vázquez-García ${ }^{\text {a }}$, M. Reyes-Pérez ${ }^{\text {a }}$, \\ J. A. Rodríguez-Ávila b , A. Espinosa-Roa ${ }^{\circ}$ \\ a Área Académica de Ciencias de la Tierra y Materiales, Universidad Autónoma del Estado de Hidalgo, 42184, Pachuca, Hidalgo, México. \\ ${ }^{b}$ Área Académica de Química, Universidad Autónoma del Estado de Hidalgo, 42184, Pachuca, Hidalgo, México. \\ ${ }^{c}$ Cátedras CONACyT - Centro de Investigaciones en Química Aplicada, 66628, Apodaca N.L., México.
}

\section{Resumen}

Se diseñó una sal orgánica a base de tris-imidazolio y ligantes quinolícos, como material atractivo para la detección de especies iónicas. Los estudios teóricos revelaron su comportamiento semiconductor. Además, las visualizaciones de los niveles de energía (HOMO y LUMO) permitieron apreciar la distribución de la densidad electrónica sobre los grupos quinolínicos y los sitios positivos que corresponden a los anillos de imidazol.

\section{Palabras Clave:}

DFT, Sal de imidazolio, Quinolina, HOMO, LUMO, Densidad electrónica.

\begin{abstract}
This paper reports the design of an organic salt based of tris-imidazolium and moieties of quinoline as an attractive material for the detection of ionic species. The theorical studies revealed its semiconducting behavior. Furthermore, the visualizations of the levels of energy (HOMO and LUMO) demonstrated the distribution of the electron density on the moieties of quinoline and the positive sites that correspond to the imidazole rings.
\end{abstract}

Keywords:

DFT, Imidazolium salt, Quinoline, HOMO, LUMO, Electron density.

\section{Introducción}

Los cálculos de química computacional han permitido con éxito el desarrollo de numerosos sensores fluorescentes (Bay et al., 2019). En este sentido, el método de la teoría del funcional de la densidad (DFT), en sus diversas formas, tiene un impacto importante como herramienta de investigación de las relaciones estructura-propiedad de moléculas y materiales, para aplicaciones específicas (Verma \& Truhlar, 2020), (Roy, 2019), (Klimes \& Michaelides, 2012), (Gidopoulos \& Wilson, 2003), (Laird et al,1996).

Los compuestos orgánicos fluorescentes son de gran importancia en el reconocimiento de especies iónicas (Erdogan, 2021). Puesto que, en algunos casos pueden llegar a interaccionar con algún analito de interés y presentar un cambio en su longitud de onda de emisión (Tanwar et al., 2020). De ahí que, las sales orgánicas fluorescentes al exhibir características físicas y electrónicas ajustables, reciban especial atención (Boydston et al., 2007). Por ejemplo, las sales de imidazolio funcionalizadas con naftalimida son prometedores sensores fluorescentes debido al mejoramiento de sus propiedades (Rizzo et al., 2020). Asimismo, a través de estudios teóricos se han dado a conocer sales de trisimidazolio funcionalizadas con antraceno como sensores selectivos de ácido pícrico, que comúnmente es usado como componente de muchos explosivos poderosos (Roy, et al., 2013).

Estudios teóricos de DFT realizados por (Vellé et al., 2017), sobre sales de imidazolio han revelado que los cationes de imidazol presentan mayor aromaticidad que en su forma no protonada. Por otra parte, derivados quinolínicos han mostrado propiedades ópticas interesantes como sensores luminiscentes (Fu, 2020), (Jiang et al., 2019), (Rams-Baron et al., 2015), (Prajapati et al., 2014). Por ello, resulta de gran interés el estudio previo de nuevas moléculas orgánicas a partir de sales de imidazolio y grupos quinolínicos.

\footnotetext{
*Autor para la correspondencia: is118581@uaeh.edu.mx

Correo electrónico: is118581@uaeh.edu.mx (Nery Islas-Rodríguez), javier_hernandez@uaeh.edu.mx (Oscar Javier Hernández-Ortiz), rosav@uaeh.edu.mx (Rosa Ángeles VázquezGarcía), mreyes@uaeh.edu.mx (Martín Reyes-Peréz) josear@uaeh.edu.mx (José Antonio Rodríguez-Ávila), arian.espinosa@ciqa.edu.mx (Arián Espinosa-Roa).
} 


\section{Metodología experimental}

Simulación computacional DFT. Se llevó a cabo, mediante el software Gaussian 09w. Para los cálculos de la energía mínima se emplearon las moléculas en fase gas aplicando la Teoría del Funcional de la Densidad (DFT) usando el modelo de densidad híbrida B3LYP (Becke, de tres-parámetros, Lee-Yang-Parr) y el conjunto base 6-31G (d,p), que comúnmente en este tipo de estudios es elegido como un compromiso entre la calidad de la estimación teórica y el alto coste computacional asociado con el alto número de dimensiones del problema para todos los átomos. Los valores energéticos de HOMO, LUMO y Band Gap fueron deducidos a partir de las estructuras estables obtenidas en la optimización. Para las visualizaciones de la distribución electrónica en los niveles, se utilizó el software Gaussian View 6.0 .

\section{Resultados y discusiones}

En este documento, se informa el diseño de una sal orgánica basada en la sal de tris-imidazolio y funcionalizada con fragmentos quinolínicos (Esquema 1). Los estudios DFT de la sal orgánica 1 previamente optimizada, revelaron que la energía del orbital ocupado más alto (HOMO) es $1.55 \mathrm{eV}$ y la energía del orbital ocupado de menor energía es (LUMO) $2.51 \mathrm{eV}$, respectivamente (Figura 1). Con un valor de Band Gap teórico de $0.96 \mathrm{eV}$. Las visualizaciones de la distribución de la densidad electrónica de las estructuras de menor energía de los orbitales HOMO y LUMO, permiten apreciar la transferencia de carga de extremo a extremo y que involucra los anillos de imidazol y los grupos quinolínicos (Figura 2).

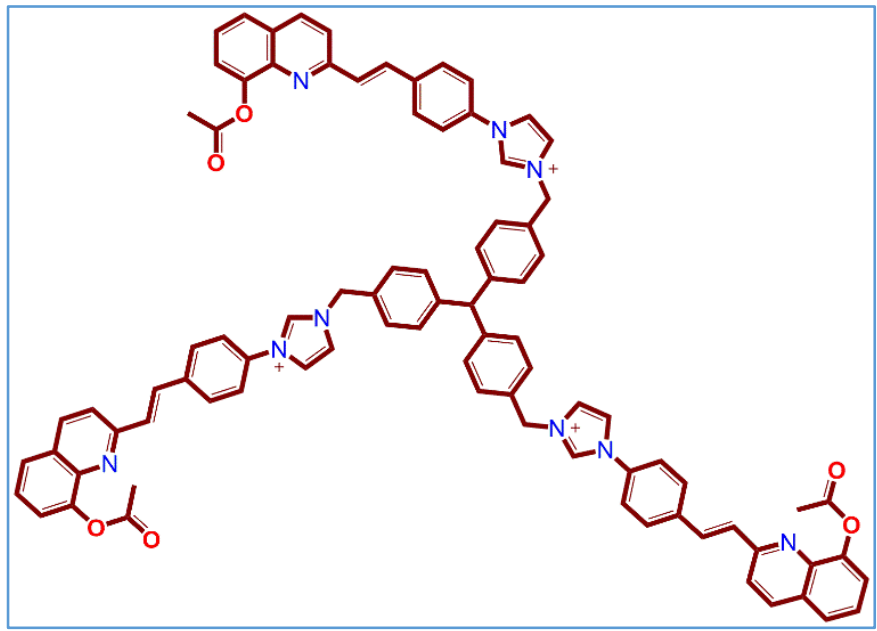

Esquema 1. Sal orgánica 1 en su forma catiónica

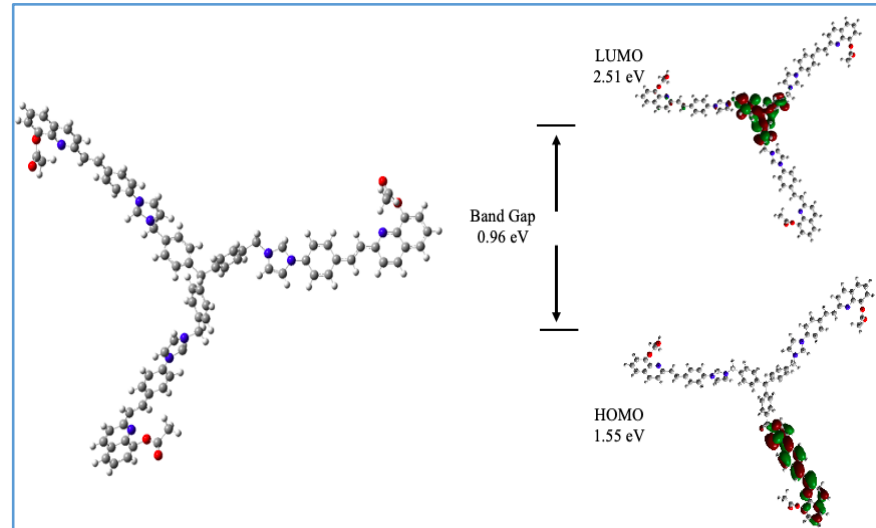

Figura 1. Visualización de la densidad electrónica de los orbitales frontera de la sal orgánica 1 en su forma catiónica y su valor de band gap teórico por DFT B3LYP/6-31G

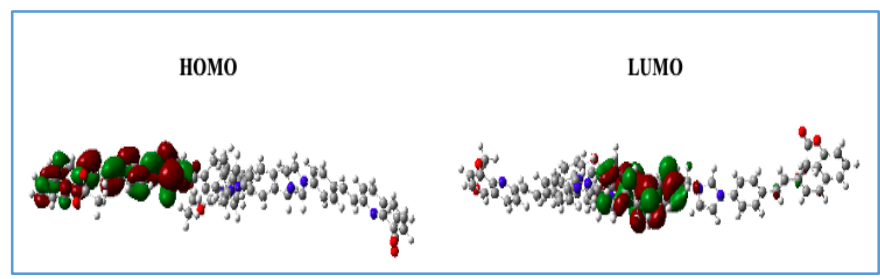

Figura 2. Visualización en plano horizontal de los niveles de energía HOMO y LUMO para la sal orgánica 1 con su geometría previamente optimizada.

Los valores de los ángulos dihedrales señalados de la macromolécula propuesta (Figura 3 ) son muy cercanos a los 0 y $180^{\circ}$ (Tabla 1), beneficiaría el grado de planaridad.

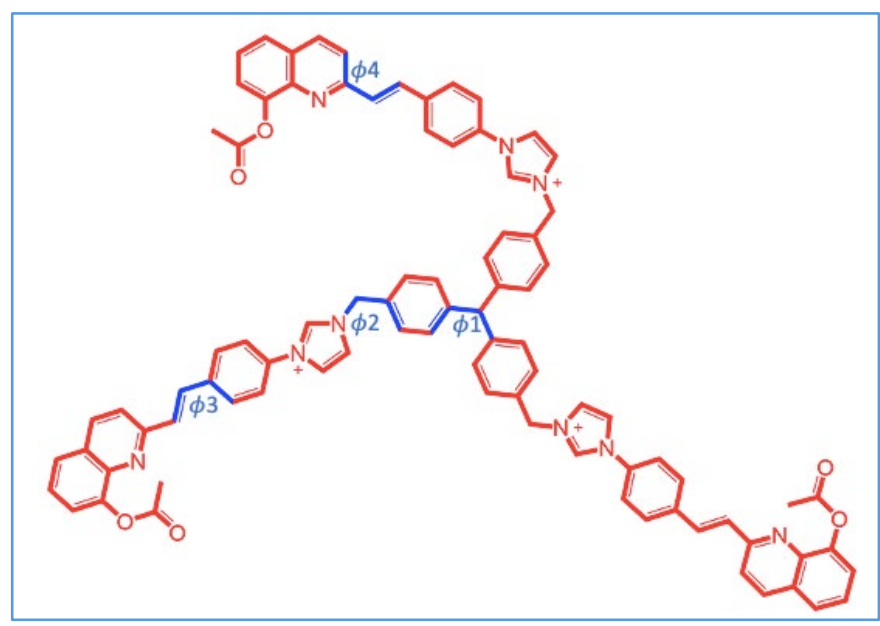

Figura 3. Estructura sal orgánica 1 y sus respectivos ángulos dihedrales señalados.

Tabla 1. Ángulos dihedrales obtenidos por DFT usando B3LYP/6-31G

\begin{tabular}{ccccc}
\hline Molécula & $\Phi_{1}\left({ }^{\circ}\right)$ & $\Phi_{2}\left({ }^{\circ}\right)$ & $\Phi_{3}\left({ }^{\circ}\right)$ & $\Phi_{4}\left(^{\circ}\right)$ \\
\hline $\begin{array}{c}\text { Sal orgánica } \\
\mathbf{1}\end{array}$ & 164.78 & 29.49 & 1.76 & 179.67 \\
\hline
\end{tabular}

Se hace la consideración del ácido pícrico para su posible detección por medio de la molécula diseñada. Por lo que se obtuvo a partir de cálculos teóricos mediante DFT del ion picrato, de sus estructuras de menor energía de los orbitales moleculares HOMO y LUMO valores de $-2.87 \mathrm{eV}$ y $0.77 \mathrm{eV}$, respectivamente y su valor de Band Gap teórico es $2.1 \mathrm{eV}$ (Figura 4). Además, se observa que el alto grado de planaridad del anión picrato permite 
que suceda la deslocalización electrónica entre su estructura molecular.

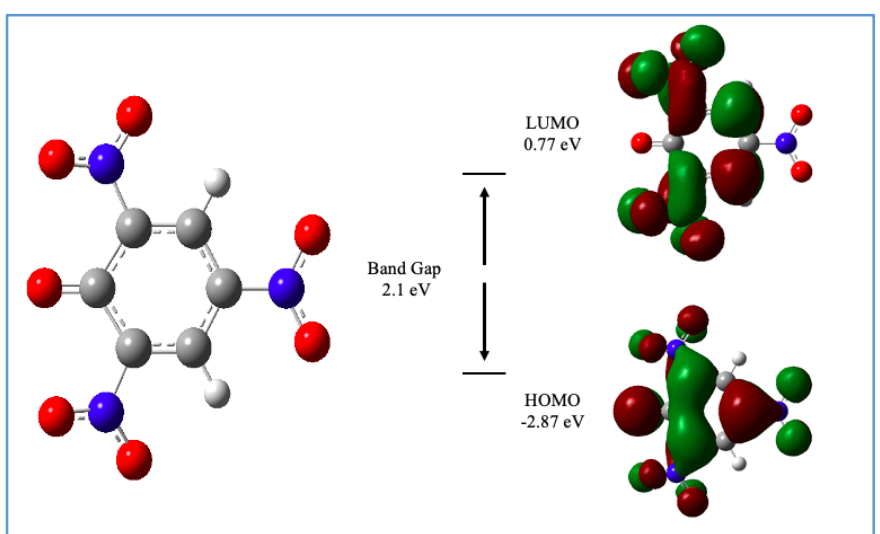

Figura 4. Visualización de la distribución de la densidad electrónica de los orbitales frontera del anión picrato y su valor de band gap teórico por DFT B3LYP/6-31G.

\section{Conclusiones}

El presente trabajo describe el diseño molecular de una nueva macromolécula orgánica fluorescente, derivada de sal de imidazolio, empleando un segmento de quinolina para su funcionalización. Los valores energéticos obtenidos de los orbitales frontera (HOMO y LUMO) de esta molécula revela su comportamiento semiconductor. Además, la distribución de la densidad electrónica sobre los anillos de imidazol cargados positivamente, favorecería la interacción electrostática con el anión picrato, como se ha reportado en otros trabajos de sales de imidazolio con aniones, por lo que se recomienda su obtención experimental para su uso potencial en el reconocimiento de especies iónicas.

\section{Agradecimientos}

Este trabajo ha sido realizado gracias a la beca otorgada por Consejo Nacional de Ciencia y Tecnología CONACyT (CVU: 593645) para estudios de Doctorado en Ciencias de los Materiales.

\section{Referencias}

Bay, M., Hien, N., Quy, P., Nam, P., Van, D., \& Quang, D. (2019). Using calculations of the electronically excited states for investigation of fluorescent sensors: A review. Vietnam Journal of Chemistry 57(4), 389400. DOI: $10.1002 /$ vjch.201900089
Boydston, A. J., Vu, P. D., Dykhno, O. L., Chang, V., Wyatt, A. R., Stockett, A. S., Ritschdorff, E. T., Shear, J. B., \& Bielawski, C. W. (2007). Modular Fluorescent Benzobis(imidazolium) Salts: Syntheses, Photophysical Analyses, and Applications. American Chemical Society 130(10), 31433156. DOI: $10.1021 / \mathrm{ja} 7102247$

Erdogan, M. (2021). A novel dibenzosuberenone bridged D-A- $\pi$-A type dye: Photophysical and photovoltaic investigations. Journal of Molecular Structure 1232, 130056. DOI: 10.1016/j.molstruc.2021.130056

$\mathrm{Fu}, \mathrm{D}$. (2020). A novel quinoline-based colorimetric fluorescent probe for hydrogen sulfide. Journal of Chemical Research 45(1-2), 76-79. DOI: $10.1177 / 1747519820924589$

Gidopoulos, N. I., \& Wilson, S. (2003). The Fundamentals of Electron Density, Density Matrix and Density Functional Theory in Atomos, Molecules and the Solid State, Progress in Theoretical Chemistry and Physics 14, 1-223. DOI: 10.1007/978-94-017-0409-0

Jiang, K., Luo, S., Pang, C., Wang, Bo., Wu, H., \& Wang, Z. (2019). A functionalized fluorochrome based on quinoline-benzimidazole conjugate: From facile design to highly sensitive and selective sensing for picric acid. Dyes and Pigments 162, 367-376. DOI: 10.1016/j.dyepig.2018.10.041

Klimes, J., \& Michaelides (2012). Perspective: Advances and challenges in treating van der Waals dispersion forces in density functional theory. The Journal of Chemical Physics 137, 120901. DOI: 10.1063/1.4754130

Laird, B. B., Ross, R. B., \& Ziegler, T. (1996). Density-Functional Methods in Chemistry: An Overview. American Chemical Society 629, 1-17. DOI: 10.1021/bk-1996-0629.ch001

Prajapati, S. M., Patel, K. D., Vekariya, R. H, Panchal, S. N., \& Patel, H. D. (2014). Recent advances in the synthesis of quinolines: a review. RSC Advance 4, 24463-24476. DOI: 10.1039/c4ra01814a

Rams-Baron, M., Dulski, M., Mrozek-Wilczklewicz, A. Korzec, M., Cieslik, W., Spaczynska, E., Bartczak, P., Ratuszna, A., Polanski, J., \& Musiol, R. (2015). Synthesis of New Styrylquinoline Cellular Dyes, Fluorescent Properties, Cellular Localization and Cytotoxic Behavior. Plos One 10(6), 1-17.DOI: 10.1371/journal.pone.0131210

Rizzo, C., Cancemi, P., Mattiello, L., Marullo, S., \& D'Anna, F. (2020). Naphthalimide Imidazolium-Based Supramolecular Hydrogels as Biomaging and Theranostic Soft Materials. ACS Applied Materials \&Interfaces 12(43), 48442-48457. DOI: 10.1021/acsami.0c17149

Roy, A. K., (2019). Anew density functional method for electronic structure calculation of atoms and molecules. Chemical Physics, 1-32.

Roy, B., Bar, A. K., Gole, B., \& Mukherjee, S. (2013). Fluorescent TrisImidazolium Sensors for Picric Acid Explosive, ACS The Journal of Organic Chemistry 78, 1306-1310. DOI: 10.1021/jo302585a

Tanwar, A. S., Meher, N., Adil, L. R., \& Iyer, P. K. (2020). Stepwise elucidation of fluorescence-based sensing mechanisms considering picric acid as a model analyte. Royal Society of Chemistry: Analyst 145, 4753. DOI: $10.1039 / \mathrm{d} 0 \mathrm{an} 00732 \mathrm{c}$

Vellé, A., Cebollada, A., Macías, R., Iglesias, M., Gil-mole, M., \& Sanz, P. J. (2017). From Imidazole toward Imidazolium Salts and N-heterocyclic Carbene Ligands: Electronic and Geometrical Redistribution. ACS Omega 2(4), 1392-1399. DOI:10.1021/acsomega.7b00138

Verma, P., \& Truhlar, D. G. (2020). Status and Challenges of Density Functional Theory. Trends in Chemistry 2(4), 302-318. DOI: 10.1016/j.trechm.2020.02.005 